\title{
Aggregation shapes of amphiphilic ring polymers: from spherical to toroidal micelles
}

\author{
Martin Jehser $^{1,2} \cdot$ Christos N. Likos $^{2}$ \\ Received: 3 January 2020 / Revised: 6 February 2020 / Accepted: 6 February 2020 / Published online: 5 March 2020 \\ (C) The Author(s) 2020
}

\begin{abstract}
The self-assembly of Janus ring polymers is studied via a coarse-grained molecular dynamics employing a bead spring model including bending rigidity contributions to the Hamiltonian. We examine the formation and the morphology of amphiphilicitydriven clusters in the system using the number density $\rho_{N}$, the temperature $T$, the fraction of solvophobic monomers $\alpha$, and the stiffness of the polymer rings $\kappa$ as control parameters. We present a quantitative analysis of several characteristics for the formed clusters of Janus rings. Measured quantities include the distribution of the cluster size $M_{C}$ and the shape of the clusters in the form of the prolate/oblate factor $Q$ and shape factors $s f$. We demonstrate Janus rings form polymorphic micelles that vary from a spherical shape, akin to that known for linear block copolymers, to a novel type of toroidal shape, and we highlight the role played by the key physical parameters leading to the stabilization of such structures.
\end{abstract}

Keywords Janus rings $\cdot$ Self-assembly $\cdot$ Simulation $\cdot$ Molecular dynamics

\section{Introduction}

Amphiphilicity is one of the most interesting physical properties of natural or artificial molecular compounds and colloids, giving rise to an enormous variety of scenarios for selfassembly and emergence of supramolecular aggregation. The term denotes the property of two regions of the macromolecular/colloidal entities that form the building blocks of the material to be physically or chemically complementary or incompatible. For instance, one of them would be attracted to the solvent and one repelled from it; or one would be soluble in one kind of two immiscible solvents and the other in the other, as is the case with surfactants with a hydrophilic and a lyophilic block.

Christos N. Likos

christos.likos@univie.ac.at

Martin Jehser

martin.jehser@univie.ac.at

1 Faculty of Chemistry, University of Vienna, Währingerstraße 42, A-1090 Vienna, Austria

2 Faculty of Physics, University of Vienna, Boltzmanngasse 5, A-1090 Vienna, Austria
A prominent example of such amphiphilic particles are chemically heterogeneous, nanosized colloids that have emerged in the last two decades as novel building blocks for steering the self-assembly, structure, dynamics, and macroscopic phase behavior in condensed matter and soft materials, also known as patchy colloids [4, 5, 23, 43, 59, 64]. These nano- to micron-sized colloidal particles possess chemically or physically decorated surfaces with selective interactions against the patched and non-patched sectors of the surfaces of other colloids. The large variety of self-assembly scenarios $[16,17,23,62,64]$ that arise from these patchy colloid interactions is also tunable through control of the anisotropy of their interaction patterns. In addition, the ability to steer the number and the (orientational) flexibility of the patches gave rise to a number of advances in our understanding of the lowtemperature phases and the critical behavior of colloidal systems with a limited number of bonding partners $[8,50,52$, 53], contributing thereby to our understanding of fundamental questions pertaining to the stability of liquids and gels at low temperatures. More ordered and disordered phases emerge when interaction anisotropy becomes combined with shape anisotropy $[14,15,58]$. Surface heterogeneities on colloidal particles can also result from the existence of heterogeneously distributed surface charges, which gives rise to the so-called inverse patchy colloids (IPCs) [11]. Whereas conventional patchy particles are realized by adding attractive patches on 
the surface of otherwise repulsive particles, IPCs have patches that electrostatically repel each other, while they attract the rest of the colloidal surface that is free of patches. The interplay between attraction and repulsion of oppositely and like charged regions results into a complex effective potential between IPCs [7] and associated, highly nontrivial self-assembly scenarios $[9,10]$.

At the opposite end of the line from the patchy colloids as far as the internal flexibility is concerned lie diblock copolymers, i.e., flexible polymer chains consisting of a solvophilic block A and a solvophobic block B. These well-studied block copolymer solutions exhibit a variety of phases at finite concentration, the most common one being a micellar phase, in which spherical aggregates (micelles) with a B-core and A-corona form [20, $26,39-41]$. A related system is associating polymers [12, 51, 56] containing units that are capable of forming reversible, physical bonds with similar units of other chains. These include van der Waals interactions, $\pi-\pi$ stacking, hydrogen bonding, and metal-ligand coordination $[2,56]$, and they cover at least two orders of magnitude in terms of binding energy, ranging from one to several hundred $\mathrm{kJ} / \mathrm{mol}$. The reversibility of the bonds leads, at high concentrations, to the formation of reconfigurable, supramolecular networks $[12,51]$.

A third category of nanosized amphiphilic particles has a hybrid nature between the hard colloids and the flexible block copolymers mentioned above. These are polymer-based nanocolloids formed by, e.g., centrally grafted block copolymers or by surface patterning by polymers of varying solvent quality. Members of this class have been termed soft patchy nanoparticles [6, 19, 21]. Examples of this class of particles can be mostly found in polymer-based systems: some characteristic examples are Janus-like polymer vesicles [61], micelles based on targeted design of functionalized dendritic polymers [47], patchy nanoparticles with tunable symmetries resulting from the self-aggregation of block copolymer chains $[19,63]$ or stars [13], block copolymer nanoparticles with microphase separation structures [27], hydrophobic dendrimers particles with dynamic hydrophobic patches [25] or colloids grafted with poor-solvent polymer brushes [19], and, lastly, polyelectrolyte brushes [3, 24, 48]. Concrete experimental realizations of such end-functionalized macromolecules are also zwitterionic polybutadiene star polymers $[44,57]$ or polybutadiene/polyisoprene block copolymer stars in selective solvents. At the infinite dilution limit (single particle state diagram), the antagonism between entropic and enthalpic contributions leads to the formation of aggregation regions (patches) for the B-type monomers on the surface of the stars. The single particle properties (patchiness, patch arrangement, angular and radial correlations, and extent) can be readily tuned by the number of arms per star, the ratio between solvophobic and solvophilic monomers, and the attraction strength [13, 34, 35, 49].

Although the combination of various polymer architectures (linear, star, dendritic) and amphiphilicity has attracted a great deal of attention, amphiphilic ring polymers have hardly been studied up to date. This is an important missing piece of the picture, as ring polymers are known to be significantly different from linear ones in a number of ways, including their overall statistical-mechanical properties [36, 42], their rheology [32], and their ability to form specific types of threading that result into glassy states in the melt $[30,37,38,54]$. Cyclic block copolymers that feature chemically distinct sequences are thus forming excellent experimental systems to study the self-assembly of block copolymer rings in finite concentration $[31,55,65]$. The purpose of this work is to conduct a number of simulations on model amphiphilic rings to explore the morphology of the resulting supramolecular aggregates and the influence of the ring architecture on the same. We discover unique signatures of the ring topology on the shape of the resulting micelles, including the formation of a novel type of toroidal micelles. Although toroidal micelles are not unknown, having already been observed for linear block copolymers, ours differ from those in two important ways: first, most of the hitherto known toroidal micelles require triblock copolymers [18, 46] (see however Ref. [29] for toroidal micelles made of diblock copolymers), as opposed to the diblock copolymer rings discussed here. And secondly, the previously discovered toroidal micelles are essentially looped cylindrical micelles, i.e., they result from a different morphology upon connecting the two ends [28], whereas in our case, the torus forms from the outset as a primary structure.

The rest of our paper is organized as follows: The model and the methods we employed for our diblock copolymer rings are presented in Sect. 2 and details on the simulation techniques in Sect. 3. We show and discuss our results in Sect. 4, and in Sect. 5, we summarize and draw our conclusions.

\section{Model and methods}

The utilized model is a microscopic monomer-resolved model for semiflexible Janus ring polymers. As shown in Fig. 1, each

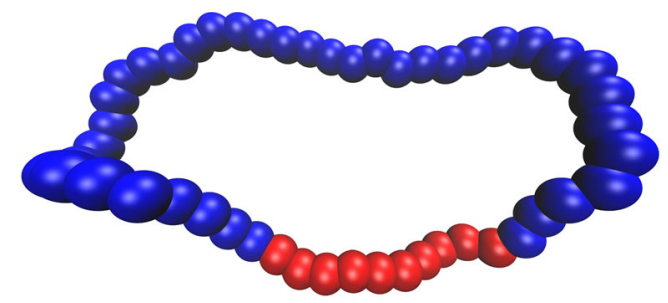

Fig. 1 Graphical representation (snapshot) of the semiflexible Janus polymer ring with $N=50$ monomers. Shown in red are the $N_{A}=10$ attractive $A$ monomers, leaving $N_{R}=40$ repulsive blue $R$ ones, respectively, representing an amphiphilicity of $\alpha=1 / 5$. The ring is a snapshot of the simulations at temperature $T=0.9$ and bending strength $\kappa=30$ 
ring consists of $N_{R}$ purely repulsive monomers $R$ and $N_{A}$ attractive monomers $\mathrm{A}$ in an $A_{N_{A}} R_{N_{R}}$ block copolymer structure with $N=N_{A}+N_{R}$ total monomers. The two different monomer species have equal mass $m$. Nonbonded monomers interact via the truncated and shifted Lennard-Jones (LJ) potential:

$U_{L J}(r)=4 \varepsilon\left[\left(\frac{\sigma}{r}\right)^{12}-\left(\frac{\sigma}{r}\right)^{6}-\left(\frac{\sigma}{r_{c}}\right)^{12}+\left(\frac{\sigma}{r_{c}}\right)^{6}\right] \Theta\left(r_{c}-r\right)(1)$

with the Heaviside step function $\Theta(x)$. Here, $\sigma$ and $\varepsilon$ denote the zero-crossing distance and the depth of the potential well of the unshifted LJ potential, respectively. The distance of monomers is given by $r$ and the cutoff distance by $r_{c}$, at which the potential is shifted to zero. There are three different nonbonded interactions possible, i.e., $A-A, R-R$, and $A-R$. The interaction of repulsive beads among themselves and with attractive monomers, $R-R$ and $R-A$, is that of the so-called Weeks-Chandler-Andersen (WCA) excluded volume potential [60] given in Eq. (2). The WCA potential is a special and purely repulsive case of the LJ potential of Eq. (1) shifted to zero and cutoff at the potential minimum at $r_{c}=2^{1 / 6} \sigma$. Attractive beads $A-A$ interact with each other via the $\mathrm{LJ}$ potential of Eq. (1) with a cutoff distance of $r_{c}=2.5$.

$U_{W C A}(r)= \begin{cases}4 \varepsilon\left[\left(\frac{\sigma}{r}\right)^{12}-\left(\frac{\sigma}{r}\right)^{6}+\frac{1}{4}\right] & \text { ifr } / \sigma<2^{1 / 6} ; \\ 0 & \text { if } r / \sigma \geq 2^{1 / 6} .\end{cases}$

In the present model, polymers are formed by bonded monomers interacting via the finitely extensible nonlinear elastic (FENE) bond potential:

$U_{F E N E}(r)=-\frac{k R_{0}^{2}}{2} \ln \left[1-\left(\frac{r}{R_{0}}\right)^{2}\right]$

Here, $R_{0}$ and $k$ denote the maximum bond extension distance and the spring constant of the bond, respectively. Together with the purely repulsive WCA potential, the FENE bonds form the bead spring model of Kremer and Grest [33]. To achieve the aforementioned semiflexibility of the polymer rings, an additional rigidity term is introduced to the system via a quadratic-cosine bending potential:

$U_{\text {bend }}(\theta)=\kappa\left(\cos \theta-\cos \theta_{0}\right)^{2}$

with $\kappa$ being the bending strength constant, $\theta$ the angle between two successive bonds, and $\theta_{0}$ the equilibrium value of the angle.

Reduced LJ units are used throughout this work setting mass $m$, distance $\sigma$, energy $\varepsilon$, and the Boltzmann constant $k_{B}$ to unity. We choose $k=30 \varepsilon / \sigma^{2}, R_{0}=1.5 \sigma$, and $\theta_{0}=2 \pi$, and depending on the simulations $\kappa$ as $[0,5,15,30,60,120] \varepsilon$ and the reduced temperatures $T^{*}$ as $[0.8,0.9,1.0,1.1] k_{B} T / \varepsilon$, whereby we simplify the notation in what follows by setting $T^{*} \rightarrow T$. With the mentioned values for $\varepsilon, \sigma, k$, and $R_{0}$, the combined potential $U_{W C A}+U_{F E N E}$ between connected monomers has a sharp minimum at $0.96 \sigma$. This, together with the steepness of $U_{W C A}$ and $U_{F E N E}$, keeps the bond distance within small fluctuations. Thus, chain uncrossability is fulfilled, preventing concatenation or knotting of the polymer rings.

\section{Simulation details}

We investigate systems with $M=250$ rings of size $N=50$ in various concentrations $\rho_{N}=[0.005,0.01,0.02,0.05,0.1]$, with $\rho_{N}=M N / V_{b o x}$ being the number density and $V_{b o x}$ the volume of the cubic simulation box. To initialize the run, the rings in the simulation box are set up as flat discs with random orientation taking special care to avoid concatenation as shown in Fig. 2. In the present work, we also vary the degree of amphiphilicity $\alpha=N_{A} / N$ in the range of $[0.12,0.5]$ for fixed $N=50$, giving us 4 tunable parameters altogether, i.e., $T, \kappa, \alpha$, and $\rho_{N}$.

To study the self-assembly of the Janus semiflexible polymer rings, we carry out constant $N V T$ Langevin dynamics simulations with implicit solvent and periodic boundary conditions. The corresponding equations of motion read as [1]:

$m \ddot{r}_{i}(t)=F_{i}(t)-\gamma m \dot{r}_{i}(t)+\eta_{i}(t)$

Here, $r_{i}$ is the position of the $i$ th monomer, $m$ its mass, and $F_{i}(t)$ the conservative force acting on particle $i$, defined as $-\nabla_{i}\left(U_{L J / W C A}+U_{F E N E}+U_{b e n d}\right)$. The quantity $\eta_{i}(t)$ is a random

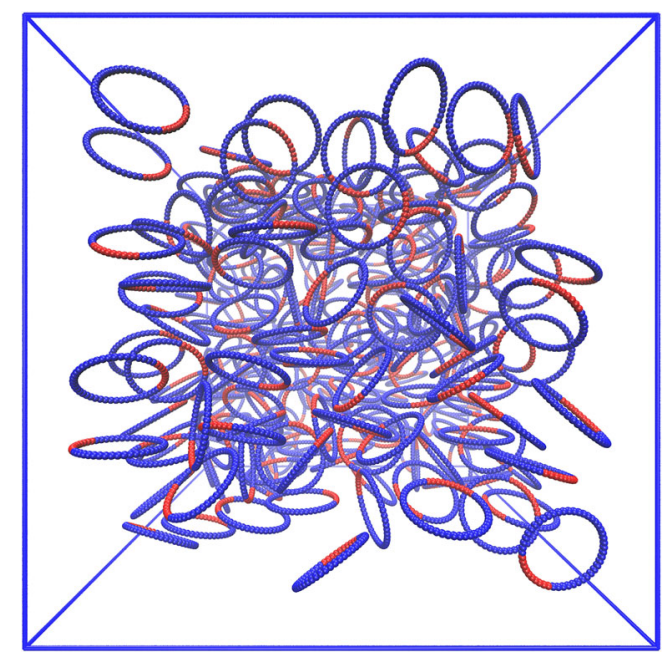

Fig. 2 Graphical representation (snapshot) of a semiflexible Janus polymer ring simulation box with $M=250$ rings and $N=50$ monomers each, representing the state at the initial system setup. Shown is an amphiphilicity $\alpha=1 / 5$ with attractive beads represented in red, whereas blue are the repulsive ones, respectively. The snapshot is taken after energy minimization of the simulation box at temperature $T=0.9$, bending strength $\kappa=30$, and number density $\rho_{N}=0.01$ 
force, with $\left\langle\eta_{i}(t)\right\rangle=0$, which is related to the friction coefficient $\gamma$ by the fluctuation dissipation relation

$\left\langle\eta_{i}^{\alpha}(t) \eta_{j}^{\beta}\left(t^{\prime}\right)\right\rangle=2 \gamma m k_{B} T \delta_{i j} \delta_{\alpha \beta} \delta\left(t-t^{\prime}\right)$

where $\alpha$ and $\beta$ denote Cartesian components. The simulations are executed with the LAMMPS [45] molecular dynamics software package. The unit of time is set by $t_{0}=\sqrt{\left(m \sigma^{2} / \varepsilon\right)}$, and the friction coefficient $\gamma$ is chosen as $10^{-2} t_{0}^{-1}$. We integrate the equations of motion with a timestep of $\Delta t=10^{-3} t_{0}$ and use $2 \times 10^{8}$ timesteps for the equilibration of the system and $1 \times 10^{9} \Delta t$ for data collection. The presented quantities are further averaged over at least five distinct simulation runs with different starting configurations and random seeds for temperature control.

We identify clusters of Janus rings by the condition of attractive beads $A$ belonging to different rings being within the cutoff distance of $r_{c}=2.5$. We study cluster size $M_{C}$, i.e., the number of rings in a cluster, and cluster shape $Q$, as functions of the mentioned parameters $T, \kappa, \alpha$, and $\rho_{N}$. We define the shape as the oblateness of the centers of mass (COM) of rings in a cluster.

$Q=\left(3 s f_{1}-1\right)\left(3 s f_{2}-1\right)\left(3 s f_{3}-1\right)$

with the shape factors $s f_{1,2,3}=e v_{1,2,3} / R_{g}^{2}$ and $e v_{1,2,3}$ being the eigenvalues of the gyration tensor (i.e., the length of the main axes of the equivalence ellipsoid) of the centers of mass, normalized by the squared radius of gyration $R_{g}^{2}$. The shape factors are defined to have the following properties: $s f_{1}<s f_{2}<s f_{3}$ and $s f_{1}+s f_{2}+s f_{3}=1$. The prolate/oblate factor $Q$ demonstrates three distinct limiting values, namely, $Q=2$ for rod-like shape, $Q=0$ for a sphere, and $Q=-1 / 4$ for a disc. The centers of mass of rings forming a perfect torus have a shape value of $Q=-1 / 4$, where all the COM are in the same plane and evenly spaced on a circle around the center of the torus, whereas a random clustering conformation would most likely be centered around $Q=0$, depending on $M_{C}$. A drawback of the prolate/oblate factor $Q$ as an indicator for torus shape stems from the fact that rings need not be equally spaced around the center of the torus. This is especially true for clusters of small $M_{C}$, leading to a more prolate shape as cases of $s f_{2} \ll s f_{3}$ become more likely, even though $s f_{1} \approx 0$. For this reason we also plot $s f_{1}$ with its limiting values between 0 and $1 / 3$.

\section{Results and discussion}

In this section, we focus on one of the variable parameters at a time, investigating aggregation number and shape of the selfassembled clusters, to form a coherent picture of the system.

\section{The influence of density}

We will start our discussion by varying the density of the system from $\rho_{N}=0.005$ to 0.100 while keeping the temperature at $T=0.9$ and the amphiphilicity at $\alpha=0.2$ and comparing the resulting findings for two different stiffnesses, i.e., fully flexible $\kappa=0$ and semiflexible $\kappa=30$ Janus rings. We see a typical snapshot of the system at density $\rho_{N}=0.005$ in Fig. 3 and at $\rho_{N}=0.1$ in Fig. 4 for the semiflexible Janus ring system. In both of the limiting cases shown in Figs. 3 and 4, one can see a clustering of attractive beads, forming the core of toroidal micelles. At first glance, the influence of density seems to only have a minor impact on the ability to form these toroids, other than being more readily recognizable with the "naked eye" due to a less crowded environment for less dense systems. We emphasize at this point that here and in what follows, we base our findings for the characteristics of the aggregation patterns on direct, "brute-force" results arising from MD simulations that develop from independent runs. We have not employed any hybrid Monte Carlo MD or smart, biased techniques to guarantee equilibrium, and accordingly, we cannot exclude that the system is not trapped to a local minimum. However, we have often observed rings exchanging the micelles to which they belong; therefore, the system possesses sufficiently rich internal dynamics.

To quantify these first impression findings of the snapshots, we take a closer look at the measurements we defined in Section 3. We will start our detailed analysis with the frequency distribution $\rho\left(M_{C}\right)$ of the cluster size $M_{C}$ as shown in Fig. 5. Starting with the fully flexible Janus rings in Fig. 5 a, one can see that even for the lowest density, a small portion of clusters with size greater than 1 exist forming a shallow plateau. With increasing density, an increase in average cluster size can be

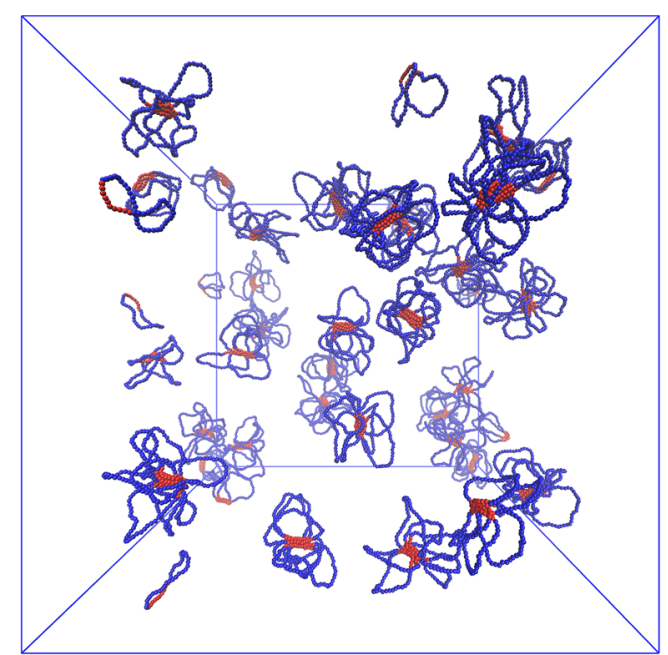

Fig. 3 Snapshot of the simulation system at $T=0.9, \alpha=0.2$, and $\kappa=30$ and density $\rho_{N}=0.005$. The blue colors represent fully repulsive beads, whereas red beads are attractive. The presented snapshot is from an equilibrated simulation run 


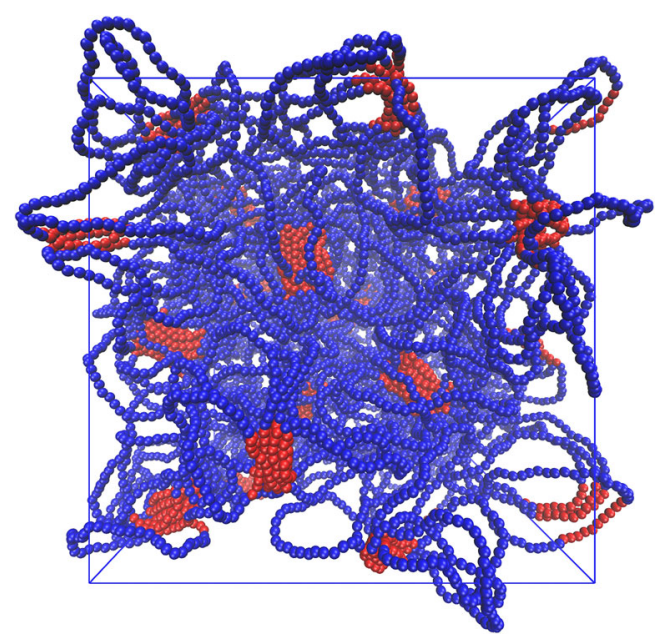

Fig. 4 Snapshot of the simulation system at $T=0.9, \alpha=0.2, \kappa=30$, and density $\rho_{N}=0.1$. The blue colors represent fully repulsive beads, whereas red beads are attractive. The presented snapshot is from an equilibrated simulation run

seen, leading to a new maximum at $M_{C} \approx 10$ for $\rho_{N}=0.01$. This second peak starts to expand and shift to higher cluster sizes with further increasing density of the system leading to $M_{C} \approx 14$ for $\rho_{N}=0.1$ at the peak maximum. An interesting find is that the largest clusters detected stay the same for all investigated densities, i.e., $M_{C-\operatorname{Max}} \approx 19$ for the fully flexible system.

The semiflexible Janus ring systems in Fig. 5 b show a different behavior. The first distinction is clearly visible for the $\rho_{N}=0.005$ system, where a sharp peak with $M_{C} \approx 7$ is formed. The proportion of free single rings is nearly zero and further decreasing with increasing number density.

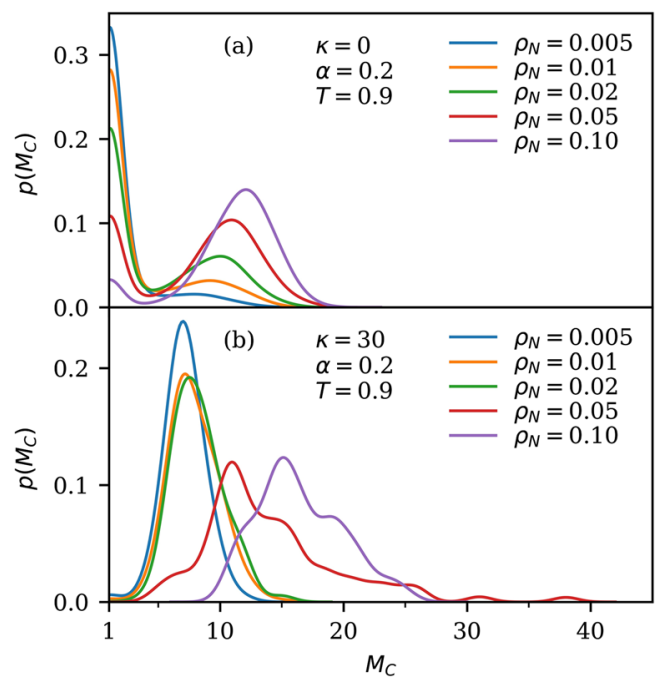

Fig. 5 Frequency distribution $\rho\left(M_{C}\right)$ of cluster size $M_{C}$ for a flexible $\kappa=$ 0 and $\mathbf{b}$ semiflexible $\kappa=30$ Janus rings, with temperature $T=0.9$ and amphiphilicity $\alpha=0.2$. The different colors represent different number densities $\rho_{N}$ as shown in the figure. Here and in all other frequency distributions shown, the raw data, obtained only for integer values of $M_{C}$, have been interpolated to produce smooth curves
Following the same overall trend to higher $M_{C}$ for increasing $\rho_{N}$ as for $\kappa=0$, the peaks shift to higher average cluster sizes. An interesting phenomenon appears for the highest two densities. The predominant single sharp peak of the lower densities changes, broadening the peak while developing distinct shoulders. This might be due to the union of micelles to bigger clusters which needs to be further investigated. The results indicate that there is a propensity of the micellar sizes to appear in integer multiples of two fundamental sizes of seven and ten rings, respectively, resulting from merging of these into new ones.

In both systems, i.e., flexible and semiflexible Janus rings, cluster formation can be found, although the semiflexible ring system shows a more complex picture. To try and understand these features better, we take a closer look at the shape of these clusters. In the simulation snapshot presented in Figs. 6 and 7, we can qualitatively compare flexible and semiflexible Janus rings. We can see that the flexible system is much more compact and looks more spherical compared to the semiflexible system. We also see the difference in the attractive centers of the clusters. The flexible Janus rings collapse their attractive beads to form densely packed spherical cores with the repulsive residue sticking out at random positions resembling the arms of a star polymer. In the case of the semiflexible Janus rings (Fig. 7), we can see a more torus-like shape of the clusters, where the attractive centers are forming a cylindrical core with the repulsive residues connecting top and bottom of these cylinders. Due to the stiff nature of the semiflexible Janus rings, the cores of the tori seem to be less shielded by the repulsive parts compared to the star-like clusters of the flexible rings.

To go into a more detailed look at the shape, we will focus on the prolate/oblate factor $\langle Q\rangle$ and the first shape factor $\left\langle s f_{1}\right\rangle$ in Fig. 8 as they were introduced at the end of Sect. 3. We

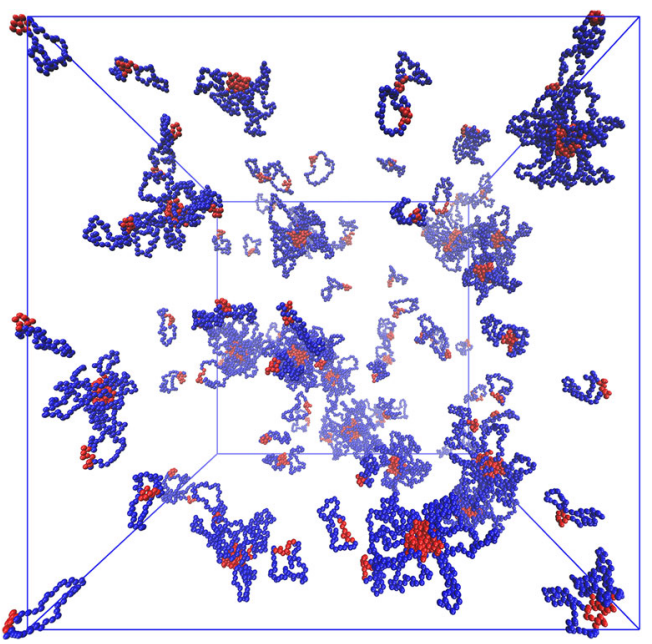

Fig. 6 Snapshot of the simulation system at $T=0.9, \alpha=0.2, \rho_{N}=0.1$, and $\kappa=0$, i.e., fully flexible Janus rings. The blue colors represent fully repulsive beads, whereas red beads are attractive. The presented snapshot is from an equilibrated simulation run 


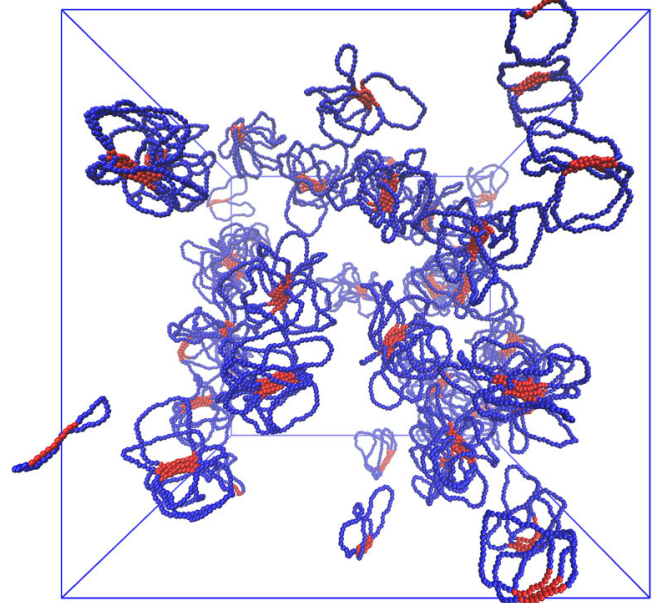

Fig. 7 Snapshot of the simulation system at $T=0.9, \alpha=0.2, \rho_{N}=0.1$, and $K=30$, i.e., semiflexible Janus rings. The blue colors represent fully repulsive beads, whereas red beads are attractive. The presented snapshot is from an equilibrated simulation run

denote ensemble averages with \langle\rangle . We start our discussion with the fully flexible system in Fig. 8 a. For cluster size three, $s f_{1}$ ("+" symbols) has to be 0 , as can be seen in the figure. With increasing cluster size, we find the earlier predicted shift to a more spherical conformation, i.e., $Q=0$ and $s f_{1}=1 / 3$. These features are independent of the density of the system in the studied range. For the semiflexible Janus rings in Fig. 8 b, we can see a clearly different picture. We start from a more prolate shape, i.e., $Q>0$, than is the case for flexible rings. For larger clusters, we tend to a more oblate shape, i.e., $Q<0$, and thus

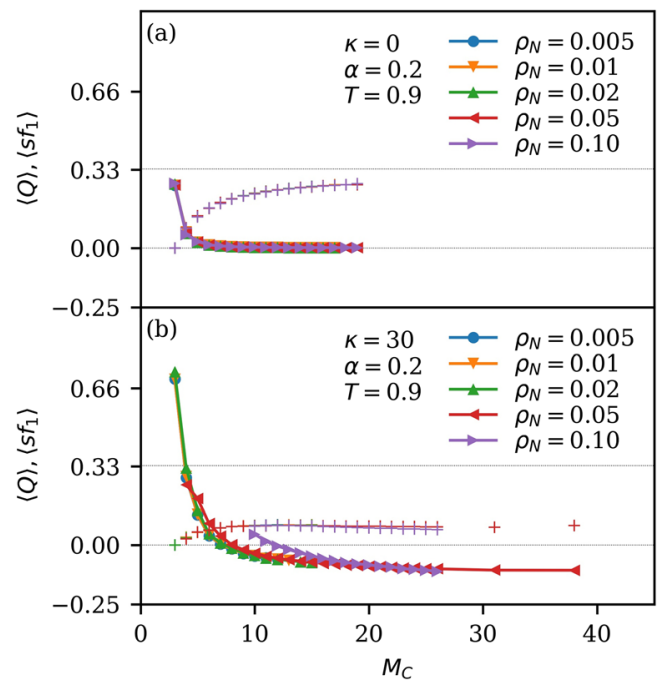

Fig. 8 Shape of COMs of rings in the same cluster in form of prolate/ oblate factor $\langle Q\rangle$ (full symbols connected with lines) and first shape factor $\left\langle s f_{1}\right\rangle$ ("+" symbol) as a function of cluster size for a flexible $\kappa=0$ and $\mathbf{b}$ semiflexible $\kappa=30$ Janus rings, with temperature $T=0.9$ and amphiphilicity $\alpha=0.2$. The different colors represent different number densities $\rho_{N}$ as shown in the figure. The dotted lines at 0.33 and 0.00 represent the limiting values of $s f_{1}$. Error bars are of the order of the symbol size and thus not explicitly shown more into the direction of a torus. This can also be seen in the values of $s f_{1} \ll 1 / 3$. Again, these features seem to be independent of the density of the system, apart from a slightly more prolate form of smaller clusters at the highest density, although $s f_{1} \ll 1 / 3$ still holds.

\section{The influence of temperature}

We will keep the number density fixed at $\rho_{N}=0.02$ for the rest of this contribution. The next issue to address is the effect of the temperature $T$ on the micelle formation of the system. We first take a look at the distribution of cluster sizes $M_{C}$ for the different temperatures as it is shown in Fig. 9. Starting with the fully flexible Janus rings in Fig. 9 a, we can find a strong dependence of cluster size on temperature. At the lowest investigated temperature, $T=0.8$, we can see clustering with mean cluster size $\left\langle M_{C}\right\rangle \approx 8$ with nearly no free single rings. With increasing $T$, the mean cluster size drops to $\left\langle M_{C}\right\rangle \approx 5$ at $T=0.9$. The peak of higher cluster sizes broadens, and the maximum shifts to larger clusters. Free rings and very small clusters become dominant. At $T=1.0$, the average cluster size has dropped to $\left\langle M_{C}\right\rangle \approx 1.8$. There are virtually no clusters of size 10 and higher detectable. At the highest investigated temperature $T=1.1$, the mean cluster size shifts to even lower values $\left\langle M_{C}\right\rangle \approx 1$.4. The largest identified clusters at this temperature where at size 9 .

The semiflexible rings are shown in Fig. 9 b. We start at the temperature $T=0.8$ where there are no free rings in the system. With increasing $T$, the mean cluster size $\left\langle M_{C}\right\rangle$ increases as well. Starting from $\left\langle M_{C}\right\rangle \approx 8$ at $T=0.8$ up to $\left\langle M_{C}\right\rangle \approx 8.8$ at $T=$ 1.1. With increasing $T$, the system starts to split in small and larger clusters. We see the main peak shift to larger cluster sizes, while at the same time, free rings and smaller clusters

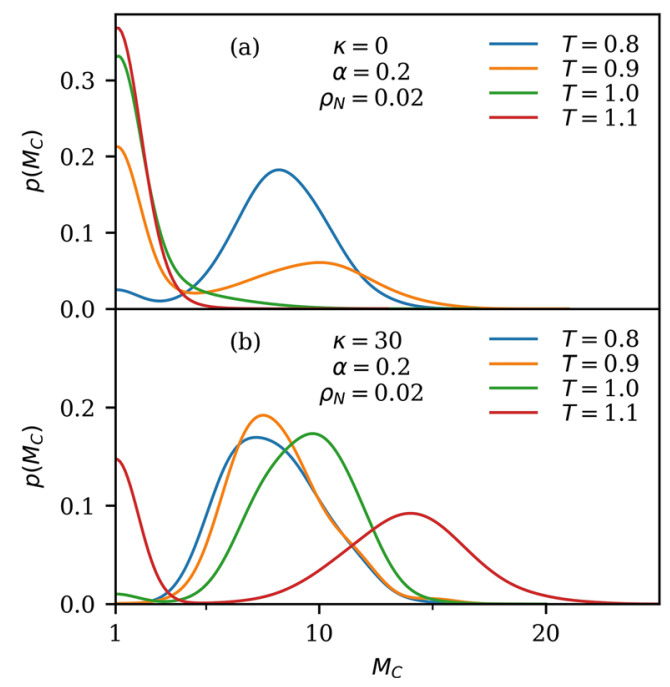

Fig. 9 Frequency distribution of cluster size $M_{C}$ for $\mathbf{a}$ flexible $\kappa=0$ and $\mathbf{b}$ stiff $\kappa=30$ Janus rings, with number density $\rho_{N}=0.02$ and degree of amphiphilicity $\alpha=0.2$. The different colors represent different temperatures $T$ as shown in the figure 
start to form. This effect seems to be the same as for the fully flexible Janus rings, albeit at higher temperatures and with a much more pronounced shift to larger cluster sizes for the semiflexible system. The stiffness of the Janus rings increases the ability to form clusters at higher temperatures.

Now let us take a look at the influence of $T$ on the shape of the clusters. In Fig. 10 a, we can study the shape of flexible ring clusters. We notice the same findings as in Fig. 8 a, i.e., an overall spherical form of the cluster with $M_{C}>5$. The more rings participating in the cluster, the more spherical the cluster becomes. Apart of this effect, the temperature seems to have no other effect on the shape of flexible clusters. The most notably distinction is that the temperature influences the maximum cluster size much stronger than the density of the system did for the fully flexible Janus rings. The effect of higher $T$ is a more instable cluster, leading to an overall smaller average cluster size and a greater portion of free single Janus rings for the fully flexible system.

The effect of temperature on the semiflexible Janus ring clusters can be seen in Fig. 10 b. We again start from a prolate shape and with increasing $M_{C}$ go to a more oblate shape. The first shape factor $s f_{1}$ is still much lower than $1 / 3$, but it increases with increasing $T$. The same is true for $Q$. With rising temperature, we go to a more and more spherical distribution of ring COMs around the center of attractive beads although still being toroidal. To illustrate this point, we present a snapshot of the highest temperature simulation for the semiflexible system rotated in a way that the central cylinder lies flat in the plane of projection in Fig. 11. One can still see the overall toroidal shape of the cluster with its central cylindrical core.

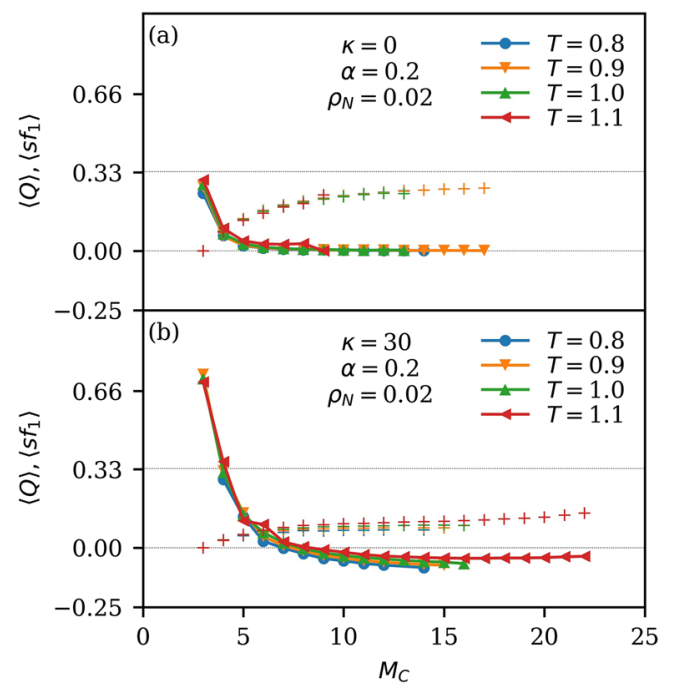

Fig. 10 Shape of COMs of rings in the same cluster in form of prolate/ oblate factor $\langle Q\rangle$ (full symbols connected with lines) and first shape factor $\left\langle s f_{1}\right\rangle$ ("+" symbol) as a function of cluster size for a flexible $\kappa=0$ and $\mathbf{b}$ semiflexible $\kappa=30$ Janus rings, with number density $\rho_{N}=0.02$ and amphiphilicity $\alpha=0.2$. The different colors represent different temperatures $T$ as shown in the figure. The dotted lines at 0.33 and 0.00 represent the limiting values of $s f_{1}$

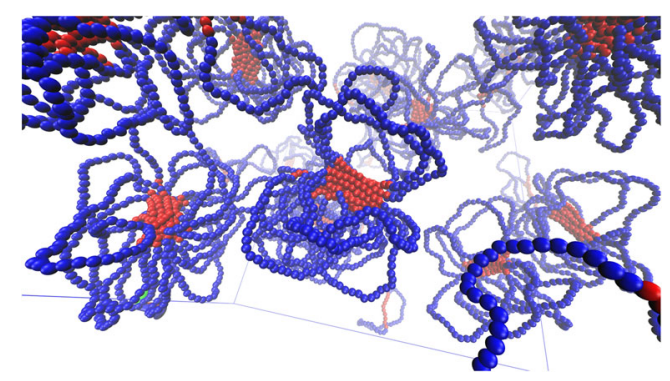

Fig. 11 Snapshot of the system at $T=1.1, \alpha=0.2, \kappa=30$, and $\rho_{N}=0.02$. The box was rotated to better see the torus shape of the cluster in the center of the picture. The blue colors represent fully repulsive beads, whereas red beads are attractive. The presented snapshot is from an equilibrated simulation run

Moreover, here and in what follows, it can be seen that the micellar core often features a tight packing of the attractive beads, a feature which, combined with rigidity, leads to visually recognizable crystalline arrangements. Though we have not attempted to perform a detailed analysis of the crystallinity of these domains, we point out that the phenomenon is common in other micelle forming systems, such as the recently investigated moderate functionality block copolymer stars [22]. It should further be mentioned that a temperature change also affects the persistence length of the rings and thus their apparent flexibility, despite the fact that $\kappa$ is kept fixed; nevertheless, the major temperature effects observed are due to the influence of the same on the relative attraction strength of the solvophobic monomers.

\section{The influence of amphiphilicity}

With temperature and density already discussed, we now take a closer look at the effects of amphiphilicity, i.e., the ratio of attractive to repulsive beads of the Janus rings. For this, we set the temperature to $T=0.9$ and the number density of the system to $\rho_{N}=0.02$. We again compare fully flexible rings $(\kappa=0)$ and semiflexible ones $(\kappa=30)$. Starting the discussion for the freely jointed Janus rings in Fig. 12 a, we can clearly see the effect of amphiphilicity on cluster formation. With the lowest amount of attractive beads $(\alpha=0.12)$, we can see no formation of clusters for the fully flexible system. For increasing amphiphilicity, we find an increase in cluster formation, with the first real emergence of clusters for values $\alpha \geq 1 / 5$. From values of $\alpha>1 / 4$ up to the highest studied ratios of $\alpha=1 / 2$, we can only see a slight increase of mean cluster size from $M_{C} \approx$ 10 to $M_{C} \approx 12$. As we will see shortly, this is a low increase compared to the semiflexible system. The surprising part here seems to be a slight non-monotonicity with the peak at $\alpha=$ 0.24 being centered around higher cluster sizes than the $\alpha=$ 0.32 one. We also can see a broadening of the peaks and the development of shoulders for the highest amphiphilicities. Interestingly, no cluster with $M_{C}>25$ can be seen for the 


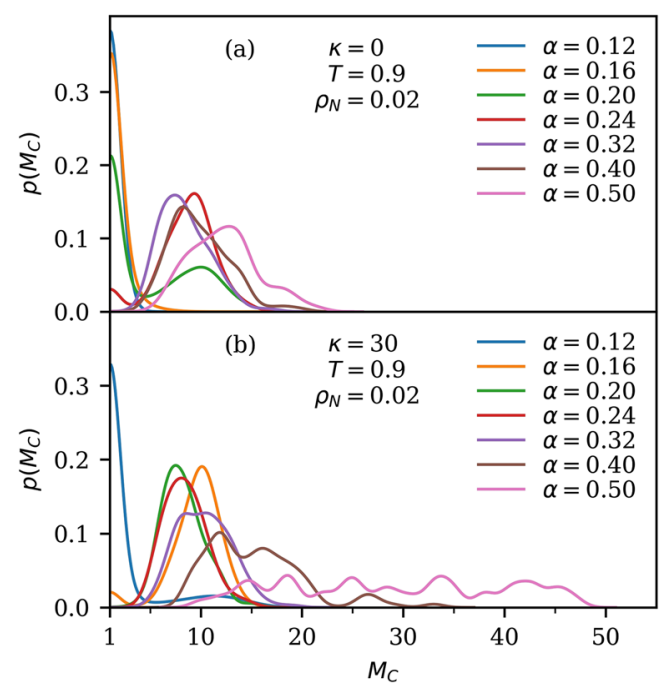

Fig. 12 Frequency distribution of cluster size $M_{C}$ for a flexible $\kappa=0$ and b stiff $\kappa=30$ rings, with temperature $T=0.9$ and number density $\rho_{N}=$ 0.02 . The different colors represent different amphiphilicity $\alpha$ as shown in the figure

flexible system regardless of $\alpha$. This might be due to shielding effects of the outward facing repulsive parts of the Janus rings.

A different picture can be seen in Fig. 12 b for the semiflexible Janus rings; where already for the lowest amphiphilicity, a small portion of clusters with size $M_{C} \approx 13$ can be found. Even for the second lowest amphiphilicity, there are virtually no single rings in the system. The peak of $\alpha=$ 0.16 is centered around the $\left\langle M_{C}\right\rangle=10$. We can see the same non-monotonic behavior as for the fully flexible system, only more pronounced. At first with increasing $\alpha,\left\langle M_{C}\right\rangle$ drops and goes to a minimum at around $\alpha=0.20$ to afterward rise again with further increasing $\alpha$, as can be seen in Fig. 13.

The second most noticeable feature of Fig. $12 \mathrm{~b}$ is the broadening of the peaks for $\alpha>1 / 4$ as was the case with the fully flexible system. The effect is strongly enhanced due to the stiffness of the Janus rings. When half of the rings beads are attractive, the formation of a torus is not the only preferred way of self-organization of the system. In Fig. 14, for example, we can

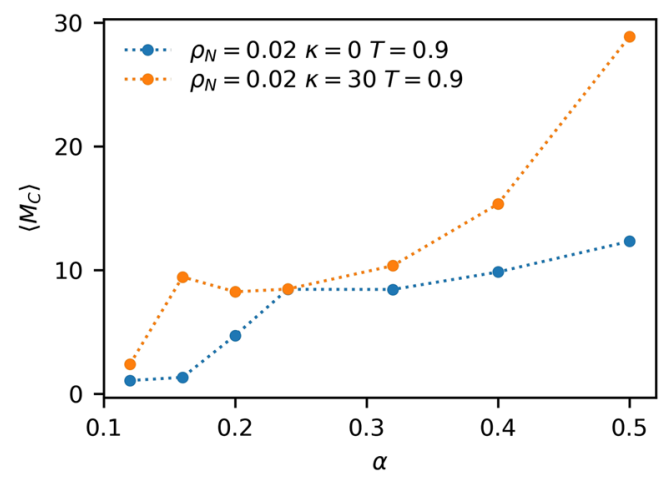

Fig. 13 Mean cluster size $\left\langle M_{C}\right\rangle$ against amphiphilicity $\alpha$. The different colors represent systems with temperature $T=0.9$ and number density $\rho_{N}=0.02$ and different $\kappa$. Fully flexible Janus rings $\kappa=0$ in blue and semiflexible $\kappa=30$ rings in orange

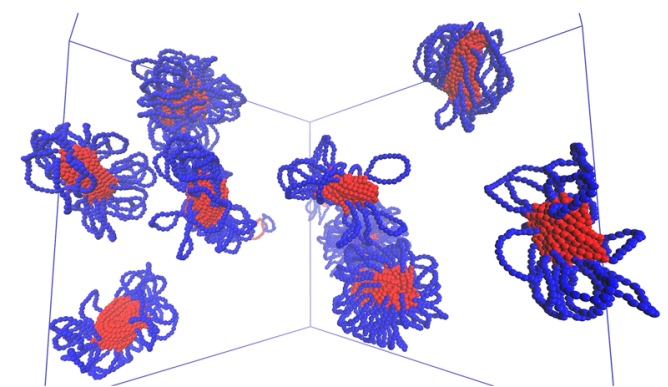

Fig. 14 Snapshot of the system at $T=0.9, \alpha=0.5, \kappa=30$, and $\rho_{N}=0.02$. The blue colors represent fully repulsive beads, whereas red beads are attractive. The presented snapshots are from equilibrated simulation runs

see a dumbbell shape and other interesting shapes as well. Due to the many attractive contacts within a cluster, the stability of clusters is very high. The many distinct values (peaks) of $M_{C}$ of the $\alpha=0.5$ system in Fig. $12 \mathrm{~b}$ are the result of clusters that, once formed, are stable throughout the whole simulation run. Due to the limited amount of simulation runs and limited rings per run, we only achieve a discrete distribution of cluster sizes $M_{C}$.

Figure 15 a shows the influence of amphiphilicity on the shape of fully flexible Janus ring clusters. Regardless of $\alpha$, all lines coincide representing the picture of a spherical distribution of COMs of rings around the attractive center for $M_{C}>5$. These are very analogous findings depicting the same picture found for the other tunable parameter for the fully flexible system. The semiflexible Janus ring system, shown in Fig. $15 \mathrm{~b}$ on the other hand, displays a very different picture. The degree of amphiphilicity has the strongest influence on shape so far discussed. For the lowest investigated value of

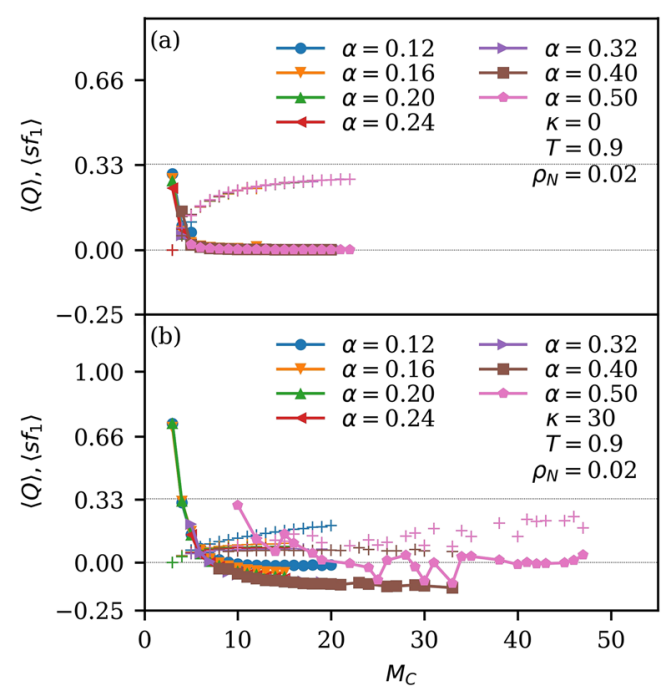

Fig. 15 Shape of COMs of rings in the same cluster in form of prolate/ oblate factor $\langle Q\rangle$ (full symbols connected with lines) and first shape factor $\left\langle s f_{1}\right\rangle$ (" + " symbol) as a function of cluster size for a flexible $\kappa=0$ and $\mathbf{b}$ semiflexible $\kappa=30$ Janus rings, with number density $\rho_{N}=0.02$ and temperatures $T=0.9$. The different colors represent different amphiphilicity $\alpha$ as shown in the figure. The dotted lines at $1 / 3$ and 0 represent the limiting values of $s f_{1}$ 
$\alpha=0.12$, we can find a very spherical conformation of COMs, although not as spherical as the fully flexible system. With increasing amphiphilicity, the clusters become more and more toroidal, i.e., decreasing values of $s f_{1}$ and $Q$. In the case of $\alpha=$ 0.4 , we reach the most toroidal conformation. For the highest amphiphilicity studied ( $\alpha=0.5)$, we can see a totally different picture. As discussed before, the torus is not the most favorable conformation of clusters with $\alpha=0.5$, as seen in Fig. 14. We can see toroidal structures, more prolate distributions of COMs, and spherical ones. A more detailed analyses of the system for $\alpha=1 / 2$ would need more simulation runs and was not part of the scope of the current contribution.

\section{The influence of stiffness}

The last variable parameter we will investigate is the stiffness of the Janus ring itself. Figure 16 shows the frequency distribution of cluster size $M_{C}$ for different values of $\kappa$. For $\kappa=0$, i.e., a fully flexible Janus ring, we can find the already discussed distribution of mostly single rings with clusters centered around size $M_{C}=10$. A slight increase of stiffness, from $\kappa=0$ to $\kappa=5$, has already a huge effect. The probability of a Janus ring to be part of a larger cluster increases drastically. There are virtually no single rings anymore, although the overall form of the peak stays the same. A further increase to $\kappa=$ 15 leads to a narrower distribution that is shifted to lower values of $M_{C}$. A further increase in stiffness does not have as high an impact on the distribution of $\mathrm{MC}$ as the steps before.

Regarding the influence of stiffness on the shape of clusters, we find $k$ to be the most influential parameter of all the investigated quantities. In Fig. 17, we can see the spherical structure of the fully flexible $(\kappa=0)$ Janus ring clusters. For the $\kappa=5$ system, we find a crossover from a spherical shape for larger $M_{C}$ to a more prolate one for lower cluster sizes like it is found for higher values of $\kappa$. A shift in shape between $\kappa=$ 5 and $\kappa=15$ can clearly be seen. With further increasing

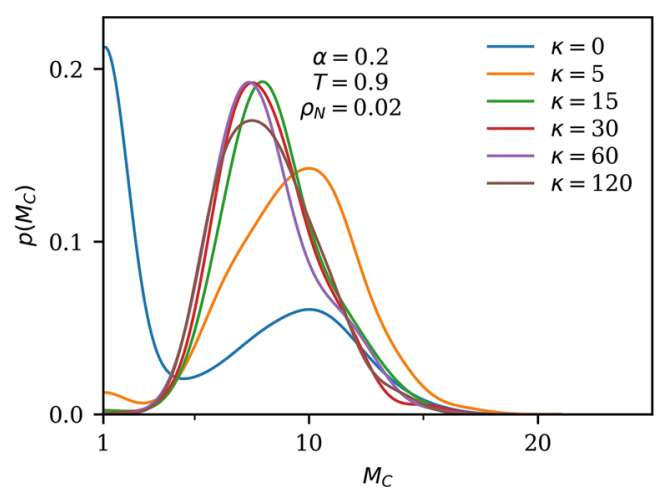

Fig. 16 Frequency distribution of cluster size $M_{C}$ for a system with temperature $T=0.9$, amphiphilicity $\alpha=0.2$, and number density $\rho_{N}=$ 0.02 . The different colors represent different values of stiffness parameter $\kappa$ as shown in the figure

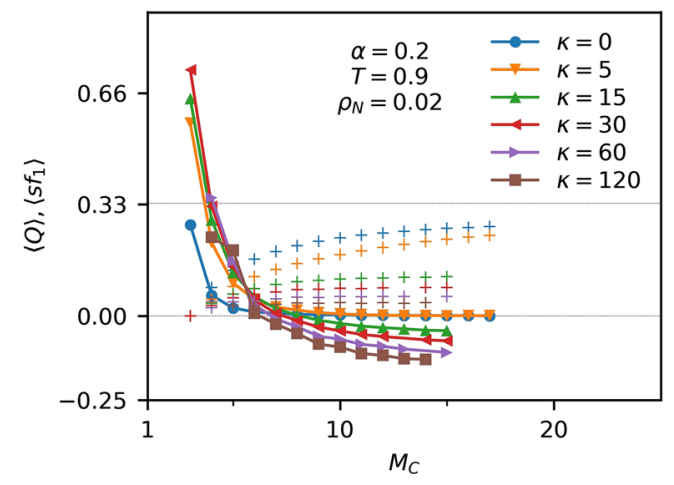

Fig. 17 Shape of COMs of rings in the same cluster in form of prolate/ oblate factor $\langle Q\rangle$ (full symbols connected with lines) and first shape factor $\left\langle s f_{1}\right\rangle$ ("+" symbol) as a function of cluster size for Janus rings with number density $\rho_{N}=0.02$, temperatures $T=0.9$, and amphiphilicity $\alpha=0.2$. The different colors represent different values of stiffness parameter $\kappa$ as shown in the figure. The dotted lines at $1 / 3$ and 0 represent the limiting values of $s f_{1}$

stiffness, the system continues to go to more and more toroidal shape of clusters. The $\kappa=120$ Janus ring system is the most toroidal of all investigated systems.

\section{Conclusion}

We have performed simulations on model amphiphilic ring polymers that contain a minority block of monomers that are solvophobic and a majority block that are soluble to a selective solvent. We have established the formation of micellar aggregates for these diblock rings, which is a common characteristic in all Janus-type mesoscopic entities in soft matter; contrary to the case of linear block copolymers, however, we have found the formation of stable toroidal micelles as primary structures arising in large parts of the parameter space spanned by the degree of amphiphilicity, the temperature, the density, and the molecular stiffness with respect to bending. We have attempted no systematic mapping out of the dependence of the critical micelle concentration or of the micellar morphologies on these parameters, due to the vastness of the parameter space. Our study serves as a starting point for such investigations, and its goal is to delineate the regions of parameter space that appear most promising for future investigations. In this spirit, we believe that future efforts should focus on more detailed investigations of the self-assembly scenarios for rings that tend to form stable toroidal micelles, i.e., rings with a high rigidity and a rather small attractive block, so that the possibility of macrophase (liquid-gas) separation be suppressed.

Acknowledgments It is a great pleasure to dedicate this paper to Matthias Ballauff on the occasion of his retirement, knowing in full that this will be only a formality and that otherwise he will remain an active researcher. Matthias, for one of us (C.N.L.), it has been a great honor to have worked with you in the past; we are looking forward to more exchanges with you also in the future! 
Funding information Open access funding provided by University of Vienna. This work has been supported by a Ph.D. Assistantship of the Faculty of Chemistry of the University of Vienna.

\section{Compliance with ethical standards}

Conflict of interest The authors declare that they have no conflict of interest.

Open Access This article is licensed under a Creative Commons Attribution 4.0 International License, which permits use, sharing, adaptation, distribution and reproduction in any medium or format, as long as you give appropriate credit to the original author(s) and the source, provide a link to the Creative Commons licence, and indicate if changes were made. The images or other third party material in this article are included in the article's Creative Commons licence, unless indicated otherwise in a credit line to the material. If material is not included in the article's Creative Commons licence and your intended use is not permitted by statutory regulation or exceeds the permitted use, you will need to obtain permission directly from the copyright holder. To view a copy of this licence, visit http://creativecommons.org/licenses/by/4.0/.

\section{References}

1. Allen M, Tildesley D (1989) Computer simulation of liquids. Oxford University Press, Oxford

2. Amin D, Likhtman AE, Wang Z (2016) Dynamics in supramolecular networks formed by associating telechelic chains. Macromolecules 49:7510-7524

3. Ballauff M (2007) Spherical polyelectrolyte brushes. Prog Polym Sci 32:1135-1151

4. Bianchi E, Blaak R, Likos CN (2011) Patchy colloids: state of the art and perspectives. Phys Chem Chem Phys 13:6397-6410

5. Bianchi E, Capone B, Colluzza I, Rovigatti L, van Oostrum P, Rovigatti L (2017) Limiting the valance: advancements and new perspectives on patchy colloids, soft functionalized nanoparticles and biomolecules. Phys Chem Chem Phys 19:19847-19868

6. Bianchi E, Capone B, Kahl G, Likos CN (2015) Soft-patchy nanoparticles: modeling and self-organization. Faraday Discuss 181:123-138

7. Bianchi E, Kahl G, Likos CN (2013) Inverse patchy colloids: from microscopic description to mesoscopic coarse-graining. Soft Matter 7:8313-8323

8. Bianchi E, Largo J, Tartaglia P, Zaccarelli E, Sciortino F (2006) Phase diagram of patchy colloids: towards empty liquids. Phys Rev Lett 97:168301

9. Bianchi E, Likos CN, Kahl G (2014) Self-assembly of heterogeneously charged particles under confinement. ACS Nano 7:4657-4667

10. Bianchi E, Likos CN, Kahl G (2014) Tunable assembly of heterogeneously charged colloids. Nano Lett 14:3412-3418

11. Bianchi E, van Oostrum P, Likos CN, Kahl G (2017) Inverse patchy colloids: synthesis, modeling and self-organization. Curr Op Colloid Interf Sci 30:8-15

12. Brunsveld L, Folmer B, Meijer EW, Sijbesma RP (2001) Supramolecular polymers. Chem Rev 101:4071-4097

13. Capone B, Coluzza I, Lo Verso F, Likos CN, Blaak R (2012) Telechelic star polymers as self-assembling units from the molecular to the macroscopic scale. Phys Rev Lett 109:238301

14. Chelakkot R, Lipowsky R, Gruhn T (2007) Novel low-density structure for hard rods with adhesive end groups. Macromolecules 39:7138-7143
15. Chelakkot R, Lipowsky R, Gruhn T (2009) Self-assembly network and bundle structures in systems of rods and crosslinkers - a Monte Carlo study. Soft Matter 5:1504-1513

16. Chen Q, Diesel E, Whitmer JK, Bae SC, Luijten E, Granick S (2011) Triblock colloids for dierected self-assembly. J Am Chem Soc 133:7725-7727

17. Chen W, Bae SC, Granick S (2011) Directed self-assembly of a colloidal kagome lattice. Nature 469:381-385

18. Chen Z, Cui H, Hales K, Li Z, Qi K, Pochan DJ, Wooley KL (2005) Unique toroidal morphology from composition and sequence contol of triblock copolymers. J Am Chem Soc 127:8592-8593

19. Chuoeiri RM, Galati E, Thérien-Aubin H, Klinkova A, Larin EM, Quejereta-Ferńandez A, Han L, Xin HL, Gang O, Zhulina EB, Rubinstein M, Kumacheva E (2016) Surface patterning of nanoparticles with polymer patches. Nature 538:79

20. Crassous JJ, Schurtenberger P, Ballauff M, Mihut AM (2015) Design of block copolymer micelles via crystallization. Polymer 62:A1-A13

21. Gârlea IC, Bianchi E, Capone B, Rovigatti L, Likos CN (2017) Hierarchical self-organization of soft patchy nanoparticles into morphologically diverse aggregates. Curr Op Colloid Interf Sci 30:1-7

22. Gârlea IC, Jaramillo Cano D, Likos CN (2019) Self-organization of gel networks formed by block copolymer stars. Soft Matter 15: $3527-3540$

23. Glotzer SC (2004) Some assembly required. Science 306:419-420

24. Guo X, Ballauff M (2001) Spherical polyelectrolyte brushes: comparison between annealed and quenched brushes. Phys Rev E 64:051406

25. Hermans MT, Broeren MAC, Gomopoulos N, van der Schoot P, van Genderen MHP, Sommerdijk NAJM, Fytas G, Meijer EW (2009) Self-assembly of soft nanoparticles with tunable patchiness. Nat Nanotechnol 4:721

26. Hickl P, Ballauff M, Jada A (1996) Small-angle X-ray contrast variation study of micelles formed by poly(styrene)-poly(ethylene oxide) block copolymers in aqueous solution. Macromolecules 29: 4006-4014

27. Higuchi T, Tajima A, Motoyoshi K, Yabu H, Shimomura M (2008) Frustrated phases of block copolymers in nanoparticles. Angew Chem Int Ed 47:8044

28. Holder SJ, Somerdijk NAJM (2011) New micellar morphologies from amphiphilic block copolymers: disks, toroids and bicontinuous micelles. Polym Chem 2:1018-1028

29. Huang H, Chung B, Jung J, Park HW, Chang T (2009) Toroidal micelles of uniform size from diblock copolymers. Angew Chem Int Ed 48:4594-4597

30. Huang Q, Ahn J, Parisi D, Chang T, Hassager O, Panyukov S, Rubinstein M, Vlassopoulos D (2019) Unexpected stretching of entangled ring macromolecules. Phys Rev Lett 122:208001

31. Iatrou H, Hadjichristidis N, Meier G, Frielinghaus H, Monkenbusch M (2002) Synthesis and characterization of model cyclic block copolymers of styrene and butadiene. Comparison of the aggregation phenomena in selective solvents with linear diblock and triblock analogues. Macromolecules 35:5426-5437

32. Kapnistos M, Lang M, Vlassopoulos D, Pyckhout-Hintzen W, Richter D, Cho D, Chang T, Rubinstein M (2008) Unexpected power-law stress relaxation of entangled ring polymers. Nat Mater 7:997-1002

33. Kremer K, Grest GS (1990) Dynamics of entangled linear polymer melts: a molecular dynamics simulation. J Chem Phys 92:5057-5086

34. Lo Verso F, Likos CN, Löwen H (2007) Computer simulation of thermally sensitive telechelic star polymers. J Phys Chem C 111:15803

35. Lo Verso F, Likos CN, Mayer C, Löwen H (2006) Collapse of telechelic star polymers into watermelon structures. Phys Rev Lett 96:187802

36. Micheletti C, Marenduzzo D, Orlandini E (2011) Polymers with spatial or topological constraints: theoretical and computational results. Phys Rep 504:1-73 
37. Michieletto D, Nahali N, Rosa A (2017) Glassiness and heterogeneous dynamics in dense solutions of ring polymers. Phys Rev Lett 119:197801

38. Michieletto D, Turner MS (2016) A topologically driven glass in ring polymers. Proc Natl Acad Sci U S A 113:5195-5200

39. Mihut AM, Chiche A, Drechsler M, Schmalz H, Di Cola E, Krausch G, Ballauff M (2009) Crystallization-induced switching of the morphology of poly(ethylene oxide)-block-polybutadiene micelles. Soft Matter 5:208-213

40. Mihut AM, Crassous JJ, Schmalz H, Drechsler M, Ballauff M (2012) Self-assembly of crystalline-coil diblock copolymers in solution: experimental phase map. Soft Matter 8:3163-3173

41. Mihut AM, Drechsler M, Moeller M, Ballauff M (2010) Spheretorod transition of micelles formed by the semicrystalline polybutadiene-block-poly(ethylene oxide) block copolymer in a selective solvent. Macromol Rapid Commun 31:449-453

42. Orlandini E, Whittington SG (2007) Statistical topology of closed curves: some applications in polymer physics. Rev Mod Phys 79: 611-642

43. Pawar AB, Kretzschmar I (2010) Fabrication, assembly, and application of patchy particles. Macromol Rapid Commun 31:150-168

44. Pitsikalis M, Hadjichristidis N, Mays JW (1996) Model mono-, diand tri- $\omega$-functionalized three-arm star polybutadienes. association behavior in dilute solution by dynamic light scattering and viscometry. Macromolecules 29:179

45. Plimpton S (1995) Fast parallel algorithms for short-range molecular dynamics. J Comput Phys 117:1-19

46. Pochan DJ, Chen Z, Cui H, Hales K, Qi K, Wooley KL (2004) Toroidal triblock copolymer assemblies. Science 306:94-97

47. Poon Z, Chen S, Engler AC, Lee H, Atas E, von Maltzahn G, Bhatia SN, Hammond PT (2010) Ligand-clustered patchy nanoparticles for modulated cellular uptake and in vivo tumor targeting. Angew Chem Int Ed 49:7266

48. Rosenfeldt S, Wittemann A, Ballauff M, Breininger E, Bolze J, Dingenouts N (2004) Interaction of proteins with spherical polyelectrolyte brushes in solution as studied by small-angle x-ray scattering. Phys Rev E:70

49. Rovigatti L, Capone B, Likos CN (2016) Soft self-assembled nanoparticles with temperature-dependent properties. Nanoscale 8: 3288-3295

50. Rovigatti L, Smallenburg F, Romano F, Sciortino F (2014) Gels of DNA nanostars never crystallize. ACS Nano 8:3567-3574

51. Seiffert S, Sprakel J (2012) Physical chemistry of supramolecular polymer networks. Chem Soc Rev 41:909-930

52. Smallenburg F, Filion L, Sciortino F (2014) Erasing no-man's land by thermodynamically stabilizing the liquid-liquid transition in tetrahedral particles. Nat Phys 10:653-657

53. Smallenburg F, Sciortino F (2013) Liquids more stable than crystals in particles with limited valence and flexible bonds. Nat Phys 9: 554-558

54. Smrek J, Chubak I, Likos CN, Kremer K (2020) Active topological glass. Nat Commun 11:26

55. Touris A, Hadjichristidis N (2011) Cyclic and multiblock polystyreneblock-polyisoprene copolymers by combining anionic polymerization and azide/alkyne "click" chemistry. Macromolecules 44: 1969-1976

56. van Ruymbeke E (2017) Preface: special issue on associating polymers. J Rheol 62:1099-1102

57. Vlassopoulos D, Pakula T, Fytas G, Pitsikalis M, Hadjichristidis N (1999) Controlling the self-assembly and dynamic response of star polymers by selective telechelic functionalization. J Chem Phys 111:1760

58. Walther A, Drechsler M, Rosenfeldt S, Harnau L, Ballauff M, Abetz V, Mueller AHE (2009) Self-assembly of Janus cylinders into hierarchical superstructures. J Am Chem Soc 131:4720-4728
59. Wang Y, Wang Y, Breed DR, Manoharan VN, Feng L, Hollingsworth AD, Weck M, Pine DJ (2012) Colloids with valence and specific directional bonding. Nature 491:51-56

60. Weeks JD, Chandler D, Andersen HC (1971) Role of repulsive forces in determining the equilibrium structure of simple liquids. J Chem Phys 54:5237-5247

61. Wurm F, Kilbinger AFM (2009) Polymeric Janus particles. Angew Chem Int Ed 48:8412

62. Yuan J, Xu Y, Walther A, Bolisetty S, Schumacher M, Schmalz H, Ballauff M, Mueller AHE (2008) Water-soluble organosilica hybrid nanowires. Nat Mater 7:718-722

63. Zhang J, Lu ZY, Sun ZY (2011) A possible route to fabricate patchy nanoparticles via self-assembly of a multiblock copolymer chain in one step. Soft Matter 7:9944-9950

64. Zhang Z, Glotzer SC (2004) Self-assembly of patchy particles. Nano Lett 4:1407-1413

65. Zhu Y, Gido SP, Iatrou H, Hadjichristidis N, Mays JW (2003) Microphase separation of cyclic block copolymers of styrene and butadiene and of their corresponding linear triblock copolymers. Macromolecules 36:148-152

Publisher's note Springer Nature remains neutral with regard to jurisdictional claims in published maps and institutional affiliations.

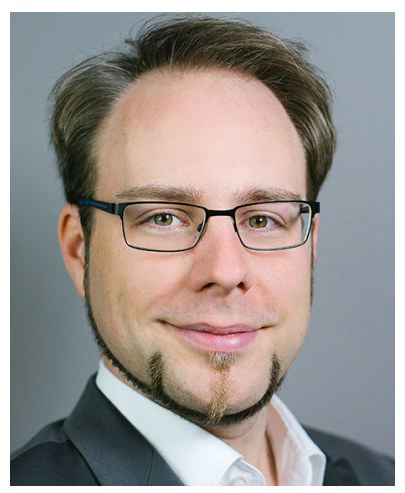

Martin Jehser studied Physical Chemistry at the University of Vienna. He obtained his Master of Science in 2014. Thereafter, Martin started his Ph.D. with Prof. Zifferer at the Faculty of Chemistry of the University of Vienna. Due to the tragic and untimely death of Prof. Zifferer in December 2015, Prof. Christos N. Likos of the Faculty of Physics of the University of Vienna became Martin's new $\mathrm{Ph}$.D. mentor. Martin is currently putting the finishing touches on his dissertation.

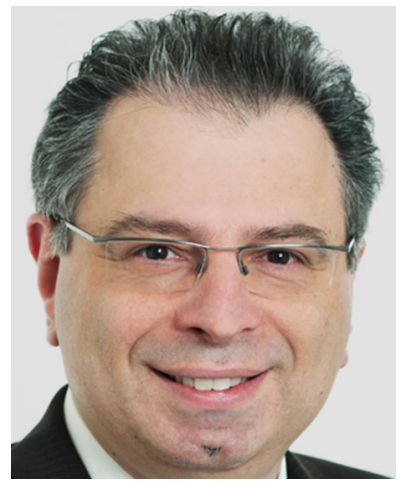

Christos Likos studied Electrical Engineering at the National Technical University of Athens. After his Diploma in 1988, he pursued graduate studies in Physics at Cornell, from where he obtained his Ph.D. in 1993. Afterward, he was Alexander von Humboldt Fellow at the University of Munich, European Communities Fellow at the University of Trieste, Research Fellow at the Jülich Research Center, and Heisenberg Fellow at the University of Cambridge. He was appointed professor of Physics at the University of Düsseldorf in 2003 and professor at the Faculty of Physics of the University of Vienna in 2010. 